\section{To: (Receiving Organization)} DISTRIBTUION
3. From: (Originating Organization) 324 AND 327 MIN SAFE

ENGINEERING

5. Proj./Prog./Dept./Div.: 300 AREA STABILIZATION

8. Originator Remarks:

$\mathrm{N} / \mathrm{A}$
6. Design Authority/Design Agent/Cog Engr: :

RS SPENCER
4. Related EDT No.

$N / A$

7. Purchase Order No.:

$N / A$

9. Equip./Component No.: $\mathrm{N} / \mathrm{A}$

10. System/Bldg./Facility: $324 \& 327$

11. Receiver Remarks: 11A. Design Baseline Document? [X] Yes [] No 324 AND 327 ESSENTIAL DRAWINGS LIST

12. Major Assm. Dwg. No.: $N / A$

13. Permit/Permit Application No.: $N / A$

14. Required Response Date: $N / A$

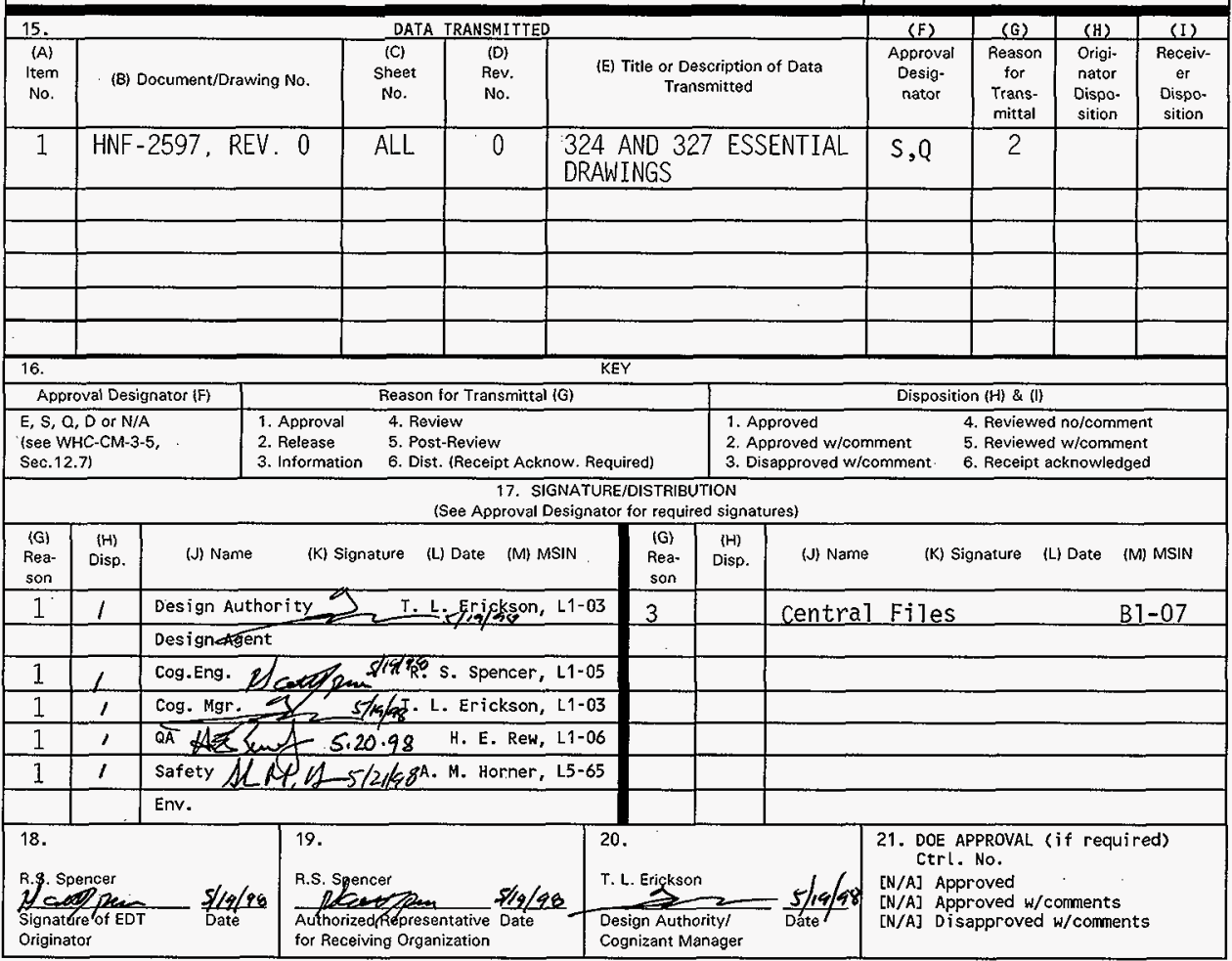


HNF-2597, Rev. 0

\title{
324 AND 327 ESSENTIAL DRAWING LIST
}

\author{
R. S. Spencer
}

B\&W Hanford Company, Richland, WA 99352

U.S. Department of Energy Contract DE-AC06-96RL13200

EDT/ECN: $\quad 623084$

Org Code: 19210

B\&R Code: EW7002010
UC: 2000

Charge Code: K4M21

Total Pages: 24

Key Words: Essential drawings, 324 and 327, Control Print File

Abstract: The purpose of this document is to publish a list of essential drawings for the 324 and 327 Facilities.

TRADEMARK DISCLAIMER. Reference herein to any specific comercial product, process, or service by trade nane, trademark, manufacturer, or otherwise, does not necessarily constitute or imply its endorsenent, recommendation, or favoring by the United States Government or any agency thereof or its contractors or subcontractors.

Printed in the United states of America. To obtain copies of this document, contact: UHC/BCS Document Control Services, P.0. Box 1970, Mailstop H6-08, Richland WA 99352, Phone (509) 372-2420; FaX (509) 376-4989.

$\frac{\text { Ravis }}{\text { Bease Aporoval }}$

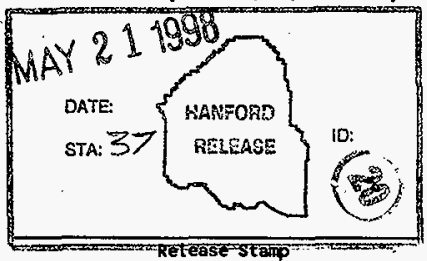




\section{Essential Drawing List}

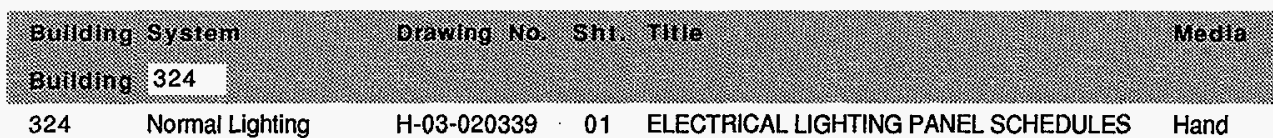

324 HVAC Controls H-03-020348 01 ELECTRICAL: ELEMENTARY DIAGRAMS PART Hand
1

324 HVAC Controls H-03-020348 02 ELECTRICAL ELEMENTARY DIAGRAMS PART Hand
1

324 HVAC Controls H-03-020349 01 ELECTRICAL ELEMENTARY DIAGRAMS PART Hand

324 HVAC Controls H-03-020350 O1 ELECTRICAL ELEMENTARY DIAGRAMS PART Hand
3

324 HVAC Controls H-03-020350 02 $\frac{3}{3}$ ELECTRICAL ELEMENTARY DIAGRAMS PART . CAD

\begin{tabular}{llll}
324 & HVAC Controls & H-03-020363 01 & $\begin{array}{l}\text { INSTRUMENTATION HEATING \& VENTILATION Hand } \\
\text { ENGINEERING DIAGRAM }\end{array}$ \\
\hline
\end{tabular}

\begin{tabular}{llllll}
\hline 324 & HVAC Controls & H-03-020365 & 01 & $\begin{array}{l}\text { Instrumentation-ElectricalVentilation Control } \\
\text { Connection Diagram and Panel Pipi }\end{array}$ & Hand \\
\hline 324 & HVAC Controls & H-03-020385 & 01 & $\begin{array}{l}\text { INSTRUMENTATION PROCESS VENTILATION } \\
\text { CONTROL DIAGRAM }\end{array}$ & Hand \\
\hline
\end{tabular}

\begin{tabular}{llllll}
\hline 324 & Fire Protection & H-03-027926 & 01 & $\begin{array}{l}\text { Fuels Recycle Pilot Plant Automatic Sprinklers } \\
\text { Fire Zone Key Plan \& Plot Plan Fire Zone III }\end{array}$ & Hand \\
\hline 324 & Fire Protection & H-03-027926 & 02 & AUTOMATIC SPRINKLERS FIRE ZONES I\& II & Hand
\end{tabular}

\begin{tabular}{llllllll}
\hline 324 & Fire Protection & H-03-027926 & 03 & $\begin{array}{l}\text { AUTOMATIC SPRINKLERS FIRE ZONE III, IV, \& } \\
\text { XIV }\end{array}$ & Hand \\
\hline 324 & Fire Protection & H-03-027926 & 04 & AUTOMATIC SPRINKLERS FIRE ZONES V \& VI & Hand \\
\hline 324 & Fire Protection & H-03-027926 & 05 & $\begin{array}{l}\text { AUTOMATIC SPRINKLERS FIRE ZONES VII \& } \\
\text { XX Hand }\end{array}$ \\
\hline 324 & Fire Protection & H-03-027926 & 06 & AUTOMATIC SPRINKLERS FIRE ZONE VIII & Hand \\
\hline 324 & Fire Protection & H-03-027926 & 07 & AUTOMATIC SPRINKLERS FIRE ZONES IX \& X & Hand \\
\hline
\end{tabular}




\section{Essential Drawing List}

\begin{tabular}{|c|c|c|c|c|c|}
\hline 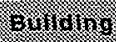 & Sisingin & 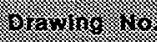 & 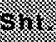 & 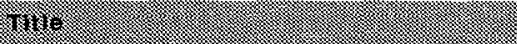 & 460 \\
\hline 324 & Fire Protection & $\mathrm{H}-03-027926$ & 08 & AUTOMATIC SPRINKLERS FIRE ZONES XI \& XII & Hand \\
\hline 324 & Fire Protection & $\mathrm{H}-03-027926$ & 09 & AUTOMATIC SPRINKLERS FIRE ZONE XIII & Hand \\
\hline 324 & Fire Alarm & $H-03-027926$ & 10 & $\begin{array}{l}\text { ELECTRICAL FIRE ALARM WIRING FOR FLOW } \\
\text { SWITCHES 1ST FLOOR \& BELOW PLAN }\end{array}$ & Hand \\
\hline
\end{tabular}

324 Normal Power H-03-028613 01 ELECTRICAL DIAGRAMS, PANEL SCHEDULES Hand

324 Normal Power $\quad$ H-03-029269 01 ELECTRICAL PANEL SCHEDULES Hand

324 Normal Power H-03-029269 02 ELECTRICAL PANEL SCHEDULES Hand

\begin{tabular}{|c|c|c|c|c|c|}
\hline 324 & Normal Power & H-03-029269 & 03 & ELECTRICAL PANEL SCHEDULES & Hand \\
\hline 324 & Normal Power & H-03-029269 & 04 & ELECTRICAL PANEL SCHEDULES & Hand \\
\hline 324 & Normal Power & H-03-029269 & 05 & ELECTRICAL PANEL SCHEDULES & Hand \\
\hline 324 & Normal Power & $\mathrm{H}-03-029269$ & 06 & ELECTRICAL PANEL SCHEDULES & Hand \\
\hline 324 & HVAC & $\mathrm{H}-03-034617$ & 01 & $\begin{array}{l}\text { DRAWING LISTHVAC HEAT RECOVERY FLOW } \\
\text { \& CONTROL DIAGRAMS }\end{array}$ & Hand \\
\hline 324 & Normal Lighting & H-03-041725 & 01 & $\begin{array}{l}\text { LIGHTING PLAN, SCHEDULES, \& ONE-LINE } \\
\text { DIAGRAM }\end{array}$ & Hand \\
\hline 324 & $\begin{array}{l}\text { Radioactive Liquid } \\
\text { Waste Sewer }\end{array}$ & $\mathrm{H}-03-043169$ & 02 & $\begin{array}{l}\text { ENGINEERING FLOW DIAGRAM INTER } \\
\text { BUILDING WASTE TRANSFER LINES }\end{array}$ & Hand \\
\hline 324 & Vacuum Air Sampling & H-03-043454 & 01 & $\begin{array}{l}\text { PIPING VACUUM AIR SAMPLING SYSTEM } \\
\text { PLAN - SECTIONS - DETAILS }\end{array}$ & Hand \\
\hline 324 & HVAC & $\mathrm{H}-03-049514$ & 01 & EXHAUST FLOW DIAGRAM ZONE 1 & CAD \\
\hline 324 & HVAC & $\mathrm{H}-03-049514$ & 02 & EXHAUST FLOW DIAGRAM ZONE 2 & CAD \\
\hline
\end{tabular}




\section{Essential Drawing List}

\begin{tabular}{|c|c|c|c|c|c|}
\hline \multicolumn{2}{|c|}{ 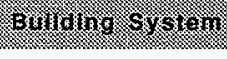 } & 8) & sin & 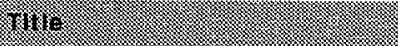 & 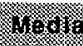 \\
\hline 324 & HVAC & H-03-049514 & 03 & EXHAUST FLOW DIAGRAM ZONE 3 & CAD \\
\hline 324 & HVAC & $\mathrm{H}-03-049514$ & 04 & EXHAUST FLOW DIAGRAM ZONE 4 & CAD \\
\hline 324 & HVAC & $\mathrm{H}-03-049514$ & 05 & EXHAAUST FLOW DIAGRAM ZONE 4 & CAD \\
\hline 324 & Steam & $\mathrm{H}-03-052464$ & 01 & STEAM \& CONDENSATE FLOW DIAC & Hand \\
\hline
\end{tabular}

\begin{tabular}{llllll}
\hline $324 \cdot$ Steam & H-03-052464 & 01 & STEAM \& CONDENSATE FLOW DIAGRAM Hand \\
\hline
\end{tabular}

324 Normal Power H-03-056548 01 ELECTRICAL PANEL SCHEDULES Hand

\begin{tabular}{|c|c|c|c|c|c|}
\hline 324 & Process Off-Gas & $H-03-070224$ & 01 & $\begin{array}{l}\text { EXHAUST FLOW DIAGRAM PROCESS OFF } \\
\text { GAS AND VESSEL VENT }\end{array}$ & CAD \\
\hline 324 & Vacuum Air Sampling & $H-03-300450$ & 01 & $\begin{array}{l}\text { PIPING-VACUUM AIR SAMPLING PLAN } \\
\text { BASEMENT AREA }\end{array}$ & CAD \\
\hline 324 & Vacuum Air Sampling & $H-03-300451$ & 01 & $\begin{array}{l}\text { PIPING-VACUUM AIR SAMPLING PARTIAL } \\
\text { PLAN } 1 S T \text { FLOOR }\end{array}$ & CAD \\
\hline 324 & Vacuum Air Sampling & $\mathrm{H}-03-300451$ & 02 & $\begin{array}{l}\text { PIPING-VACUUM AIR SAMPLING PARTIAL } \\
\text { PLAN 1ST FLOOR }\end{array}$ & CAD \\
\hline 324 & Vacuum Air Sampling & $H-03-300452$ & 01 & $\begin{array}{l}\text { PIPING-VACUUM AIR SAMPLING PLAN 2ND } \\
\text { FLOOR }\end{array}$ & CAD \\
\hline 324 & Vacuum Air Sampling & $H-03-300453$ & 01 & $\begin{array}{l}\text { PIPING/ELECTRICAL VACUUM AIR SAMPLING } \\
\text { PLAN 3RD FLOOR }\end{array}$ & CAD \\
\hline 324 & $\begin{array}{l}\text { Retention Process } \\
\text { Sewer }\end{array}$ & $H-03-300601$ & 01 & RPS PIPING BASEMENT ISOMETRIC & CAD \\
\hline 324 & $\begin{array}{l}\text { Retention Process } \\
\text { Sewer }\end{array}$ & $\mathrm{H}-03-300601$ & 02 & $\begin{array}{l}\text { RPS PIPING FIRST FLOOR PARTIAL PLAN \& } \\
\text { ELEV }\end{array}$ & CAD \\
\hline 324 & $\begin{array}{l}\text { Retention Process } \\
\text { Sewer }\end{array}$ & $\mathrm{H}-03-300601$ & 03 & RPS PIPING FIRST FLOOR ISOMETRIC & CAD \\
\hline 324 & $\begin{array}{l}\text { Retention Process } \\
\text { Sewer }\end{array}$ & $H-03-300601$ & 04 & $\begin{array}{l}\text { RPS PIPING 1ST FLOOR GALLERIES } \\
\text { ISOMETRIC }\end{array}$ & CAD \\
\hline 324 & $\begin{array}{l}\text { Retention Process } \\
\text { Sewer }\end{array}$ & $\begin{array}{l}\mathrm{H}-03-300601 \\
\end{array}$ & 05 & RPS PIPING 2ND FLOOR PLAN \& DETAILS & CAD \\
\hline
\end{tabular}




\section{Essential Drawing List}

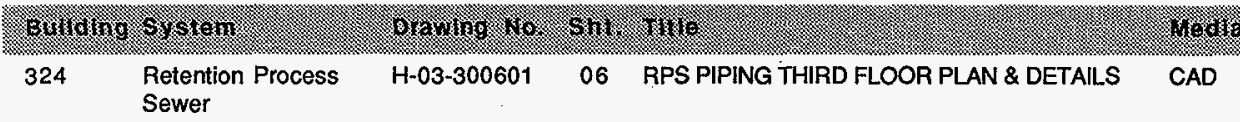

$324^{\circ} \begin{aligned} & \text { Retention Process } \\ & \text { Sewer }\end{aligned}$ H-03-300601 07. RPS PIPING BASEMENT ISOMETRIC DETAILS CAD

\begin{tabular}{llllll}
\hline 324 & $\begin{array}{l}\text { Retention Process } \\
\text { Sewer }\end{array}$ & H-03-300601 & 08 & RPS PIPING DETAILS & CAD \\
\hline 324 & $\begin{array}{l}\text { Radioactive Liquid } \\
\text { Waste Sewer }\end{array}$ & H-03-300602 & 01 & $\begin{array}{l}\text { RLWS BASEMENT \& UNDERGROUND } \\
\text { ISOMETRIC }\end{array}$ & CAD \\
\hline
\end{tabular}

324 Radioactive Liquid H-03-300602 02 RLWS/CBWS PIPING FIRST FLOOR ISOMETRIC CAD
Waste Sewer

\begin{tabular}{|c|c|c|c|c|c|}
\hline 324 & $\begin{array}{l}\text { Radioactive Liquid } \\
\text { Waste Sewer }\end{array}$ & $\mathrm{H}-03-300602$ & 03 & $\begin{array}{l}\text { RLWS/CRIB PIPING SECOND \& THIRD FLOOR } \\
\text { PLANS }\end{array}$ & CAD \\
\hline 324 & $\begin{array}{l}\text { Radioactive Liquid } \\
\text { Waste Sewer }\end{array}$ & H-03-300602 & 04 & RLWS BASEMENT ISOMETRIC DETAILS & CAD \\
\hline 324 & Process Sewer & $H-03-300603$ & 01 & $\begin{array}{l}\text { PROCESS SEWER PIPING BASEMENT } \\
\text { ISOMETRIC }\end{array}$ & CAD \\
\hline 324 & Process Sewer & $\mathrm{H}-03-300603$ & 02 & PS PIPING FIRST FLOOR ISOMETRIC & CAD \\
\hline 324 & Process Sewer & $H-03-300603$ & 03 & PS PIPING FIRST FLOOR ISOMETRIC & CAD \\
\hline 324 & Process Sewer & $\mathrm{H}-03-300603$ & 04 & PS PIPING FIRST FLOOR ISOMETRIC & CAD \\
\hline 324 & Process Sewer & $H-03-300603$ & 05 & $\begin{array}{l}\text { PROCESS SEWER PIPING SECOND FLOOR } \\
\text { ISOMETRIC \& DETAILS }\end{array}$ & CAD \\
\hline 324 & Process Sewer & $H-03-300603$ & 06 & $\begin{array}{l}\text { PROCESS SEWER PIPING THIRD FLOOR } \\
\text { ISOMETRIC \& DETAILS }\end{array}$ & CAD \\
\hline 324 & Process Sewer & $\mathrm{H}-03-300603$ & 07 & $\begin{array}{l}\text { PROCESS SEWER BASEMENT ISOMETRIC } \\
\text { DETAILS }\end{array}$ & CAD \\
\hline 324 & Process Sewer & $\mathrm{H}-03-300603$ & 08 & $\begin{array}{l}\text { PROCESS SEWER BASEMENT ISOMETRIC } \\
\text { DETAIL }\end{array}$ & CAD \\
\hline 324 & Process Sewer & $\mathrm{H}-03-300603$ & 09 & $\begin{array}{l}\text { PROCESS SEWER PIPING THIRD FLOOR } \\
\text { DETAILS }\end{array}$ & CAD \\
\hline
\end{tabular}




\section{Essential Drawing List}

\begin{tabular}{|c|c|c|c|c|c|}
\hline EIII) & s. & 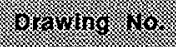 & 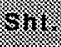 & (1) & 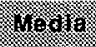 \\
\hline 324 & Sanitary Sewer & $\mathrm{H}-03-300606$ & 01 & $\begin{array}{l}\text { SANITARY PIPING FIRST FLOOR AND } \\
\text { BASEMENT ISOMETRIC }\end{array}$ & CAD \\
\hline 324 & Sanitary Sewer & $\mathrm{H}-03-300606$ & 02 & $\begin{array}{l}\text { SANITARY PIPING SECOND \& THIRD FLOOR } \\
\text { ISOMETRICS }\end{array}$ & CAD \\
\hline 324 & Sanitary Sewer & $H-03-300606$ & 03 & SANITARY PIPING ISOMETRIC DETAILS & CAD \\
\hline 324 & Roof Drain & $\mathrm{H}-03-300608$ & 02 & VTR \& DOWNSPOUT ROOF PIPING DETAILS & CAD \\
\hline 324 & Simplified & $H-03-301001$ & 01 & SIMPLIFIED BUILDING PLAN - BASEMENT & CAD \\
\hline 324 & Simplified & $\mathrm{H}-03-301002$ & 01 & SIMPLIFIED BUILDING PLAN - FIRST FLOOR & CAD \\
\hline 324 & Simplified & H-03-301.003 & 01 & SIMPLIFIED BUILDING PLAN - SECOND FLOOR & CAD \\
\hline 324 & Simplified & H-03-301004 & 01 & SIMPLIFIED BUILDING PLAN - THIRD FLOOR & CAD \\
\hline 324 & Simplified & $H-03-301005$ & 01 & SIMPLIFIED ROOF PLAN & CAD \\
\hline 324 & $\begin{array}{l}\text { Passive Fire } \\
\text { Protection }\end{array}$ & H-03-301063 & 01 & PASSIVE FIRE PROTECTION, BASEMENT & CAD \\
\hline 324 & $\begin{array}{l}\text { Passive Fire } \\
\text { Protection }\end{array}$ & $H-03-301064$ & 01 & PASSIVE FIRE PROTECTION, 1ST FLOOR & CAD \\
\hline 324 & $\begin{array}{l}\text { Passive Fire } \\
\text { Protection }\end{array}$ & $\mathrm{H}-03-301065$ & 01 & PASSIVE FIRE PROTECTION, 2ND FLOOR & CAD \\
\hline 324 & $\begin{array}{l}\text { Passive Fire } \\
\text { Protection }\end{array}$ & $H-03-301066$ & 01 & PASSIVE FIRE PROTECTION, 3RD FLOOR & CAD \\
\hline 324 & HVAC & $H-03-301067$ & 01 & HVAC BASEMENT AREA 1 COMPOSITE & CAD \\
\hline 324 & HVAC & $H-03-301075$ & 01 & HVAC BASEMENT AREA 3 COMPOSITE & CAD \\
\hline 324 & HVAC & $H-03-301083$ & 01 & HVAC BASEMENT AREA 4 COMPOSITE & $C A D$ \\
\hline
\end{tabular}




\section{Essential Drawing List}

\section{Buriting 58 \& 1 .}

$324 \cdot$ HVAC
8.

$\mathrm{H}-03-301091 \quad 01$ HVAC BASEMENT AREA 5 COMPOSITE inedo:

CAD

\begin{tabular}{llllllll}
\hline 324 & HVAC & H-03-301099 & 01 & HVAC 1ST FLOOR AREA 1 COMPOSITE & CAD \\
\hline 324 & HVAC $:$ & H-03-301107 & 01 & HVAC 1ST FLOOR AREA 2 COMPOSITE & CAD \\
\hline 324 & HVAC & H-03-301115 & 01 & HVAC 1ST FLOOR AREA 3 COMPOSITE & CAD \\
\hline
\end{tabular}

324 HVAC H-03-301123 01 HVAC 1ST FLOOR AREA 4 COMPOSITE CAD

\begin{tabular}{llllllll}
\hline 324 & HVAC & H-03-301131 & o1 & HVAC 1ST FLOOR AREA 5 COMPOSITE & CAD \\
\hline 324 & HVAC & H-03-301139 & O1 & HVAC 1ST FLOOR AREA 6 Composite & CAD &
\end{tabular}

324 HVAC H-03-301147 01 HVAC 1ST FLOOR AREA 7 Composite CAD

324 HVAC H-03-301155 01 HVAC 1ST FLOOR AREA 8 Composite

\begin{tabular}{llllllll}
\hline 324 & HVAC & H-03-301163 & 01 & HVAC 2nd FLOOR AREA 1 Composite & CAD \\
\hline 324 & HVAC & H-03-301171 & 01 & HVAC 2nd FLOOR AREA 2 Composite & CAD \\
\hline 324 & HVAC & H-03-301179 & 01 & HVAC 2nd FLOOR AREA 3 Composite & CAD \\
\hline 324 & HVAC & H-03-301187 & 01 & HVAC 2nd FLOOR AREA 4 Composite & CAD \\
\hline 324 & HVAC & H-03-301195 & 01 & HVAC 2nd FLOOR AREA 5 Composite & CAD \\
\hline 324 & HVAC & H-03-301203 & 01 & HVAC 2nd FLOOR AREA 6 Composite & CAD \\
\hline 324 & HVAC & H-03-301211 & 01 & HVAC 2nd FLOOR AREA 7 Composite & CAD \\
\hline
\end{tabular}




\section{Essential Drawing List}

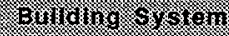

324 HVAC
3)

H-03-301219
01

HVAC

\section{mo}

(1)

3rd FLOOR AREA 1 Composite

H-03-301227 01 HVAC 3rd FLOOR AREA 3 Composite

CAD

H-03-301235 01 HVAC 3rd FLOOR AREA 4 Composite

CAD

324 HVAC

H-03-301243

01

HVAC 3rd FLOOR AREA 5 Composite

CAD

324 HVAC

$324 \quad$ HVAC

H-03-301251

01

HVAC 3rd FLOOR AREA 7 Composite

CAD

\begin{tabular}{|c|c|c|c|c|c|}
\hline 324 & HVAC & $H-03-301259$ & 01 & HVAC Roof Plan AREA 1 Composite & CAD \\
\hline 324 & HVAC & $H-03-301267$ & 01 & HVAC Roof Plan AREA 2 Composite & CAD \\
\hline 324 & HVAC & $\mathrm{H}-03-301275$ & 01 & HVAC Roof Plan AREA 3 Composite & CAD \\
\hline 324 & HVAC & H-03-301283 & 01 & HVAC Roof Plan AREA 4 Composite & CAD \\
\hline 324 & HVAC & H-03-301299 & 01 & HVAC Roof Plan AREA 6 Composite & CAD \\
\hline 324 & HVAC & H-03-301315 & 01 & HVAC Roof Plan AREA 8 Composite & CAD \\
\hline 324 & Normal Power & H-03-301323 & 01 & $\begin{array}{l}\text { POWER ONE-LINE DIAGRAM DRAWING } \\
\text { DIRECTORY }\end{array}$ & CAD \\
\hline 324 & Normal Power & $H-03-301323$ & 02 & POWER ONE-LINE DIAGRAM & CAD \\
\hline 324 & Normal Power & H-03-301323 & 03 & POWER ONE-LINE DIAGRAM & CAD \\
\hline 324 & Normal Power & $\mathrm{H}-03-301323$ & 04 & POWER ONE-LINE DIAGRAM & CAD \\
\hline 324 & Normal Power & $\mathrm{H}-03-301323$ & 05 & POWER ONE-LINE DIAGRAM & CAD \\
\hline
\end{tabular}




\section{Essential Drawing List}

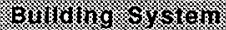

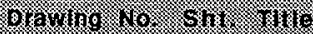

.

324 Normal Power

$\mathrm{H}-03-301323$

06 POWER ONE-LINE DIAGRAM

$C A D$

\begin{tabular}{lllllll}
\hline 324 & Normal Power & H-03-301323 & 07 & POWER ONE-LINE DIAGRAM & CAD \\
\hline 324 & Normal Power & H-03-301323 & 08 & POWER ONE-LINE DIAGRAM & CAD \\
\hline 324 & Normal Power & H-03-301323 & 09 & POWER ONE-LINE DIAGRAM & CAD \\
\hline 324 & Normal Power & H-03-301323 & 10 & POWER ONE-LINE DIAGRAM & CAD \\
\hline
\end{tabular}

324 Normal Power H-03-301323 11 POWER ONE-LINE DIAGRAM 480 VOLT PANEL CAD

\begin{tabular}{lllll}
\hline 324 & Normal Power & H-03-301323 & 12 & POWER ONE-LINE DIAGRAM
\end{tabular}

324 Normal Power H-03-301323 13 POWER ONE-LINE DIAGRAM 48OV PANELS CAD

324 Normal Power H-03-301323 14 POWER ONE-LINE DIAGRAM 48OV PANELS CAD

324 Normal Power H-03-301323 15 POWER ONE-LINE DIAGRAM 48OV PANELS CAD

\begin{tabular}{lllllll}
\hline 324 & Normal Power & H-03-301323 & 16 & POWER ONE-LINE DIAGRAM 480V PANELS & CAD \\
\hline 324 & Normal Power & $H-03-301323$ & 17 & $\begin{array}{l}\text { POWER ONE-LINE DIAGRAM 480V BUS } \\
\text { DUCTS }\end{array}$ & CAD \\
\hline 324 & Normal Power & H-03-301323 & 18 & $\begin{array}{l}\text { POWER ONE-LINE DIAGRAM 480 VOLT } \\
\text { PANELS }\end{array}$ & CAD \\
\hline 324 & Normal Power & H-03-301323 & 19 & $\begin{array}{l}\text { POWER ONE-LINE DIAGRAM 480 VOLT } \\
\text { PANELS }\end{array}$ & CAD \\
\hline 324 & Normal Power & H-03-301323 & 20 & $\begin{array}{l}\text { POWER ONE-LINE DIAGRAM 480 VOLT } \\
\text { PANELS }\end{array}$ & CAD \\
\hline 324 & Fire Alarm & H-03-301501 & 01 & $\begin{array}{l}\text { ELECTRICAL. FIRE ALARM SYSTEM } \\
\text { BASEMENT PLAN }\end{array}$ & CAD \\
\hline
\end{tabular}




\section{Essential Drawing List}

\begin{tabular}{|c|c|c|c|c|c|}
\hline Enging & a) & 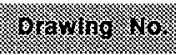 & (3) & 36 & 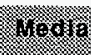 \\
\hline 324 & Fire Alarm & $\mathrm{H}-03-301501$ & 02 & $\begin{array}{l}\text { ELECTRICAL FIRE ALARM SYSTEM FIRST } \\
\text { FLOOR PLAN }\end{array}$ & $C A D$ \\
\hline 324 & Fire Alarm & $H-03-301501$ & 03 & $\begin{array}{l}\text { ELECTRICAL FIRE ALARM SYSTEM SECOND } \\
\text { FLOOR PLAN }\end{array}$ & CAD \\
\hline 324 & Fire Alarm & $\mathrm{H}-03-301501$ & 04 & $\begin{array}{l}\text { ELECTRICAL FIRE ALARM SYSTEM 3RD } \\
\text { FLOOR PLAN }\end{array}$ & $C A D$ \\
\hline 324 & Fire Alarm & $H-03-301501$ & 05 & $\begin{array}{l}\text { ELECTRICAL FIRE ALARM SYSTEM OFFICE } \\
\text { TRAILERS }\end{array}$ & CAD \\
\hline 324 & Fire Alarm & $H-03-301501$ & 06 & $\begin{array}{l}\text { ELECTRICAL FIRE ALARM SYSTEM CONT } \\
\text { PANEL WIRING DIAG }\end{array}$ & $C A D$ \\
\hline 324 & Fire Alarm & $H-03-301501$ & 07 & $\begin{array}{l}\text { ELECTRICAL FIRE ALARM SYSTEM CONT } \\
\text { PANEL WIRING DIAG }\end{array}$ & CAD \\
\hline 324 & Fire Alarm & $\mathrm{H}-03-301501$ & 08 & $\begin{array}{l}\text { ELECTRICAL FIRE ALARM SYSTEM WIRING } \\
\text { DIAGRAM }\end{array}$ & CAD \\
\hline 324 & Normal Power & $\mathrm{H}-03-302835$ & 01 & ELECTRICAL PANEL SCHEDULES & CAD \\
\hline 324 & Criticality Alarm & $H=03-303049$ & 01 & ALARMS BASEMENT AREA 1 CRITICALITY & $\mathrm{CAD}$ \\
\hline 324 & Criticality Alarm & $\mathrm{H}-03-303059$ & 01 & ALARMS BASEMENT AREA 3 CRITICALITY & $\mathrm{CAD}$ \\
\hline 324 & Criticality Alarm & $H-03-303069$ & 01 & ALARMS BASEMENT AREA 4 CRITICALITY & $\mathrm{CAD}$ \\
\hline 324 & Criticality Alarm & $\mathrm{H}-03-303089$ & 01 & ALARMS 1ST FLOOR AREA 1 CRITICALITY & CAD \\
\hline 324 & Criticality Alarm & $H-03-303099$ & 01 & ALARMS $1 S T$ FLOOR AREA 2 CRITICALITY & CAD \\
\hline 324 & Criticality Alarm & $\mathrm{H}-03-303109$ & 01 & ALARMS 1ST FLOOR AREA 3 CRITICALITY & CAD \\
\hline 324 & Criticality Alarm & $\mathrm{H}-03-303119$ & 01 & ALARMS 1 ST FLOOR AREA 4 CRITICALITY & $\mathrm{CAD}$ \\
\hline 324 & Criticality Alarm & H-03-303129 & 01 & ALARMS 1ST FLOOR AREA 5 CRITICALITY & $C A D$ \\
\hline
\end{tabular}




\section{Essential Drawing List}

\begin{tabular}{|c|c|c|c|c|c|}
\hline \multicolumn{2}{|c|}{ Bulloging o s stern } & 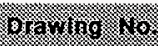 & S3in & (3) & .10018 \\
\hline 324 & Criticality Alarm & H-03-303139 & 01 & ALARMS 1ST FLOOR AREA 6 CRITICALITY & CAD \\
\hline 324 & Criticality Alarm & H-03-303149 & 01 & ALARMS 1ST FLOOR AREA 7 CRITICALITY & $\mathrm{CAD}$ \\
\hline 324 & Criticality Alarm & $H-03-303159$ & 01 & ALARMS IST FLOOR AREA 8 CRITICALITY & CAD \\
\hline 324 & Criticality Alarm & $H-03-303169$ & 01 & ALARMS 2ND FLOOR AREA I CRITICALITY & CAD \\
\hline 324 & Criticality Alarm & $\mathrm{H}-03-303179$ & 01 & ALARMS 2ND FLOOR AREA 2 CRITICALITY & CAD \\
\hline 324 & Criticality Alarm & $H-03-303189$ & 01 & ALARMS 2 ND FLOOR AREA 3 CRITICALITY & CAD \\
\hline
\end{tabular}

\begin{tabular}{lllllll}
\hline 324 & Criticality Alarm & H-03-303219 & 01 & ALARMS 2ND FLOOR AREA 6 CRITICALITY & CAD \\
\hline 324 & Criticality Alarm & H-03-303249 & 01 & ALARMS 3RD FLOOR AREA 3 CRITICALITY & CAD
\end{tabular}

\begin{tabular}{lllllll}
\hline 324 & Criticality Alarm & $H-03-303259$ & 01 & ALARMS 3RD FLOOR AREA 4 CRITICALITY & CAD & Criticality Alarm \\
\hline 324 & $\mathrm{H}-03-303287$ & 01 & $\begin{array}{l}\text { ALARMS RF/OUTSIDE WALL PLAN } \\
\text { COMPOSITE }\end{array}$ & CAD \\
\hline
\end{tabular}

\begin{tabular}{llllllll}
\hline 324 & Criticality Alarm & $\mathrm{H}-03-303300$ & 01 & ALARMS ANNUNCIATOR PANEL COMPOSITE & CAD \\
\hline 324 & Sanitary Water & $\mathrm{H}-03-303357$ & 01 & Water Piping Basement Area 1 Composite & CAD \\
\hline 324 & Sanitary Water & $\mathrm{H}-03-303362$ & 01 & Water Piping Basement Area 3 Composite & CAD \\
\hline 324 & Sanitary Water & $\mathrm{H}-03-303367$ & 01 & Water Piping Basement Area 4 Composite & CAD \\
\hline 324 & Sanitary Water & $\mathrm{H}-03-303372$ & 01 & Water Piping Basement Area 5 Composite & CAD \\
\hline 324 & Process Water & $\mathrm{H}-03-303377$ & 01 & Water Piping 1st Floor Area 1 Composite & CAD \\
\hline
\end{tabular}




\section{Essential Drawing List}

\section{Burtaling S Y stant \\ 324 Sanitary Water

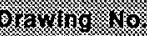 \\ H-03-303382 \\ Solit \\ 01 Water Piping 1st Floor Area 2 Composite \\ Heat \\ CAD}

324 Sanitary Water : $\quad \mathrm{H}-03-303387$ o1 Water Piping 1st Floor Area 3 Composite $\quad$ CAD

\begin{tabular}{llllllll}
\hline 324 & Sanitary Water & H-03-303402 & 01 & Water Piping 1st Floor Area 6 Composite & CAD \\
\hline 324 & Process Water & H-03-303407 & 01 & Water Piping 1st Floor Area 7 Composite & CAD
\end{tabular}

\begin{tabular}{lllllllll}
\hline 324 & Sanitary Water & H-03-303412 & 01 & Water Piping 1st Floor Area 8 Composite & CAD \\
\hline 324 & Sanitary Water & H-03-303417 & 01 & Water Piping 2nd Floor Area 1 Composite & CAD
\end{tabular}

324 Sanitary Water $\quad$ H-03-303427 01 Water Piping 2nd Floor Area 3 Composite $\quad$ CAD

324 Process Water H-03-303432 $01 \quad$ Water Piping 2nd Floor Area 4 Composite $\quad$ CAD

\begin{tabular}{llllllll}
\hline 324 & Process Water & $\mathrm{H}-03-303437$ & $\mathrm{O1}$ & Water Piping 2nd Floor Area 5 Composite & CAD \\
\hline 324 & Sanitary Water & $\mathrm{H}-03-303442$ & 01 & Water Piping 2nd Floor Area 6 Composite & CAD \\
\hline 324 & Process Water & $\mathrm{H}-03-303447$ & 01 & Water Piping 2nd Floor Area 7 Composite & CAD \\
\hline 324 & Sanitary Water & H-03-303457 & 01 & Water Piping 3rd Floor Area 3 Composite & CAD \\
\hline 324 & Sanitary Water & H-03-303462 & 01 & Water Piping 3rd Floor Area 4 Composite & CAD \\
\hline 324 & Compressed Air & H-03-303517 & 01 & Air Piping Basement Area 1 Composite & CAD \\
\hline
\end{tabular}




\section{Essential Drawing List}

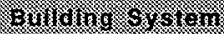

324 Compressed Air

\section{$9 \sqrt{2} \times 10198,10 \%$.}

$\mathrm{H}-03-303525$
01 Air Piping Basement Area 3 Composite
Nivola

$C A D$

324 Instrument Air $\quad$ H-03-303533 01 Air Piping Basement Area 4 Composite $\quad$ CAD

324. Compressed Air H-03-303541 01 Air Piping Basement Area 5 Composite CAD

\begin{tabular}{llllll}
\hline 324 & Compressed Air & H-03-303549 & 01 & Air Piping 1st Floor Area 1 Composite & CAD \\
\hline
\end{tabular}

324 Compressed Air H-03-303549 02 Air Piping 1st Floor Area 1 Composite

CAD

\begin{tabular}{llllll}
\hline 324 & Compressed Air & H-03-303557 & 01 & Air Piping 1st Floor Area 2 Composite & CAD
\end{tabular}

\begin{tabular}{|c|c|c|c|c|c|}
\hline 324 & Compressed Air & $H-03-303565$ & 01 & Air Piping 1st Floor Area 3 Composite & CAD \\
\hline 324 & Compressed Air & H-03-303573 & 01 & Air Piping 1st Floor Area 4 Composite & CAD \\
\hline 324 & Compressed Air & $\mathrm{H}-03-303573$ & 02 & Air Piping 1st Floor Area 4 Composite & CAD \\
\hline 324 & Compressed Air & $\mathrm{H}-03-303581$ & 01 & Air Piping 1st Floor Area 5 Composite & CAD \\
\hline 324 & Compressed Air & $\mathrm{H}-03-303581$ & 02 & Air Piping 1st Floor Area 5 Composite & CAD \\
\hline 324 & Compressed Air & $\mathrm{H}-03-303597$ & 01 & Air Piping 1st Floor Area 7 Composite & CAD \\
\hline 324 & Compressed Air & $\mathrm{H}-03-303605$ & 01 & Air Piping 1st Floor Area 8 Composite & CAD \\
\hline 324 & Compressed Air & $\mathrm{H}-03-303613$ & 01 & Air Piping 2nd Floor Area 1 Composite & CAD \\
\hline 324 & Compressed Air & $H-03-303629$ & 01 & Air Piping 2nd Floor Area 3 Composite & CAD \\
\hline 324 & Compressed Air & $\mathrm{H}-03-303629$ & 02 & Air Piping 2nd Floor Area 3 Composite & CAD \\
\hline
\end{tabular}




\section{Essential Drawing List}

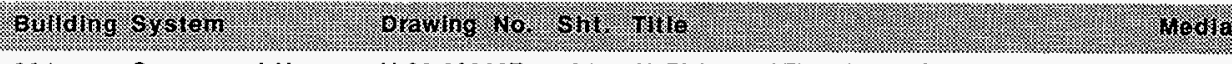

324 Compressed Air H-03-303637 01 Air Piping 2nd Floor Area 4 Composite

H-03-303645 01 Air Piping 2nd Floor Area 5 Composite

CAD

\begin{tabular}{lllllll}
\hline 324 & Compressed Air & H-03-303661 & 01 & Air Piping 2nd Floor Area 7 Composite & CAD & \\
\hline
\end{tabular}

\begin{tabular}{llllllll}
\hline 324 & Compressed Air & H-03-303669 & 01 & Air Piping 3rd Floor Area 1 Composite & CAD \\
\hline 324 & Compressed Air & H-03-303677 & 01 & Air Piping 3rd Floor Area 3 Composite & CAD \\
\hline
\end{tabular}

324. Compressed Air . H-03-303685 01 Air Piping 3rd Floor Area 4 Composite CAD.

\begin{tabular}{llllllll}
\hline 324 & Compressed Air & H-03-303685 & 02 & Air Piping 3rd Floor Area 4 Composite & CAD \\
\hline 324 & Compressed Air & H-03-303693 & 01 & Air Piping 3rd Floor Area 5 Composite & CAD
\end{tabular}

324 Compressed Air H-03-303693 01 Air Piping 3rd Floor Area 5 Composite $\quad$ CAD

324 Compressed Air H-03-303701 01 Air Piping 3rd Floor Area 7 Composite CAD

324 Steam H-03-303744 01 JET STATION PIPING ELEVATION \& DETAILS CAD

\begin{tabular}{llllll}
\hline 324 & Simplified & H-03-304231 & 01 & SIMPLIFIED BUILDING PLAN & CAD \\
\hline
\end{tabular}

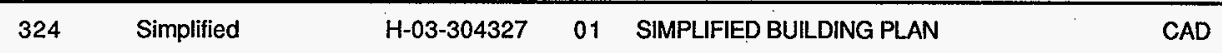

\begin{tabular}{llllllll}
\hline 324 & Normal Power & H-03-304348 & 01 & POWER ONE-LINE DIAGRAM DD48OV PANELS CAD & \\
\hline 324 & Stack Monitoring & H-03-304358 & 01 & ALARMS STACK AREA COMPOSITE & CAD \\
\hline 324 & Steam & H-03-307628 & 01 & Steam and Condensate Piping Service Tunnel & CAD \\
\hline 324 & Steam & H-03-307628 & 02 & $\begin{array}{l}\text { Steam and Condensate Piping Mechanical Rm } \\
317\end{array}$ & CAD \\
\hline
\end{tabular}




\section{Essential Drawing List}

HNF-2597 Rev.0

\begin{tabular}{|c|c|c|c|c|c|}
\hline Butating & s. & $8036 \ln _{0} \cdot 90$ & $8 x$ & 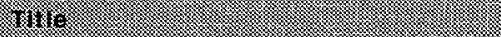 & 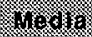 \\
\hline 324 & Steam & $H-03-307628$ & 03 & $\begin{array}{l}\text { Steam and Condensate Piping Mechanical Rm } \\
317\end{array}$ & CAD \\
\hline 324 & Steam & H-03-307628 & 04 & Steam and Condensate Piping High Bay & CAD \\
\hline
\end{tabular}


324/327 Engineering

B\&W Hanford Company

Richland, Wa

\section{Essential Drawing List}

HNF-2597 Rev.0

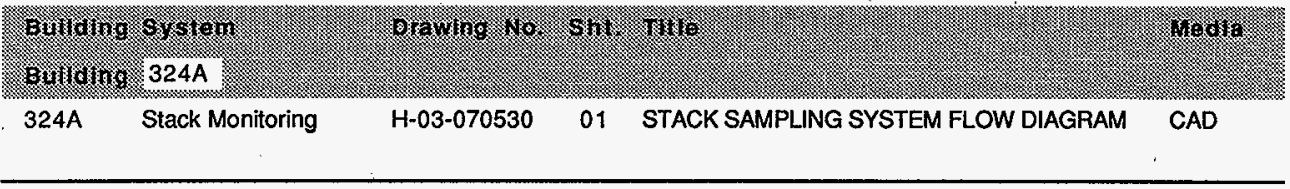




\section{Essential Drawing List}

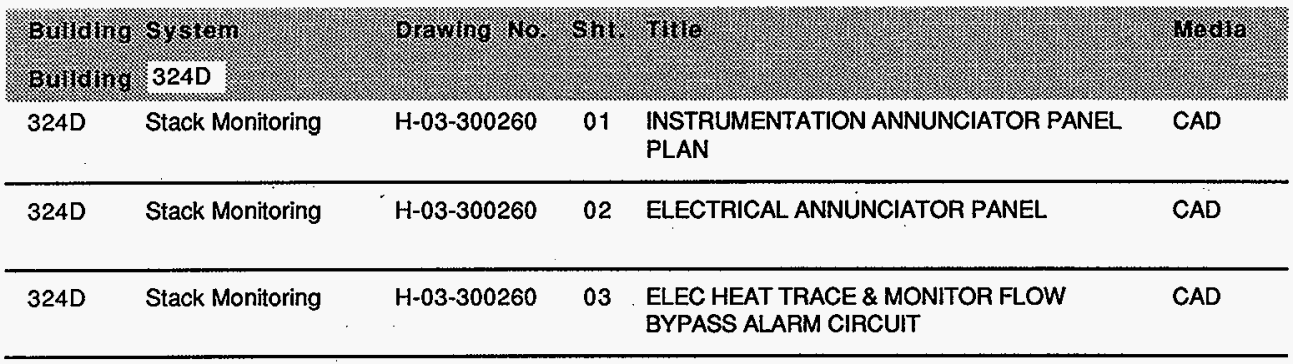




\section{Essential Drawing List}

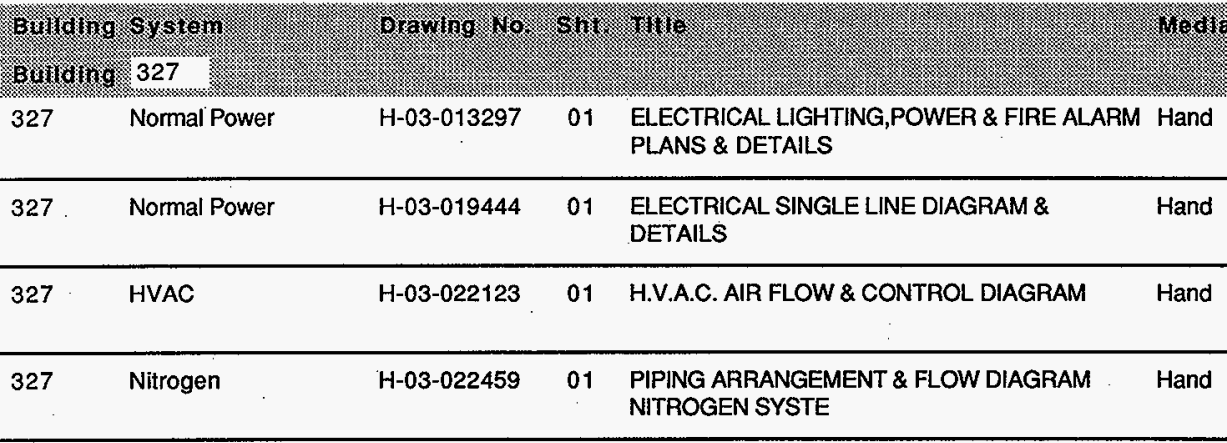

327 HVAC H-03-022545 01 HVAC FLOW SHEET \& SCHEDULES Hand

327 Sanitary Water H-03-022691 01 PLUMBING PLANS DIAGRAMS \& SECTIONS Hand

\begin{tabular}{|c|c|c|c|c|c|}
\hline 327 & Simplified & $H-03-026610$ & 01 & $\begin{array}{l}\text { SIMPLIFIED BUILDING LAYOUT FIRST FLOOR } \\
\text { PLAN }\end{array}$ & CAD \\
\hline 327 & Șimplified & $H-03-026610$ & 02 & $\begin{array}{l}\text { SIMPLIFIED BUILDING LAYOUT BASEMENT } \\
\text { FLOOR PLAN. }\end{array}$ & CAD \\
\hline 327 & Fire Protection & $\mathrm{H}-03-027700$ & 01 & $\begin{array}{l}\text { FIRE PROTECTION SPRINKLER SYSTEM } \\
\text { BASEMENT PLAN }\end{array}$ & Hand \\
\hline 327 & Fire Protection & $\mathrm{H}-03-027701$ & 01 & $\begin{array}{l}\text { FIRE PROTECTION SPRINKLER SYSTEM } \\
\text { FIRST FLOOR PLAN }\end{array}$ & Hand \\
\hline 327 & Fire Protection & $\mathrm{H}-03-027702$ & 01 & $\begin{array}{l}\text { FIRE PROTECTION SPRINKLER SYSTEM } \\
\text { FIRST FLOOR PLAn }\end{array}$ & Hand \\
\hline 327 & Normal Power & $\mathrm{H}-03-027809$ & 01 & MAIN SWITCHGEAR LAYOUT \& ELEM DIAGram & Hand \\
\hline 327 & HVAC & $\mathrm{H}-03-029314$ & 01 & AIR FLOW DIAGRAM SUPPLY AIR & Hand \\
\hline 327 & HVAC & $\mathrm{H}-03-029314$ & 02 & AIR FLOW DIAGRAM SUPPLY AIR & Hand \\
\hline 327 & Nitrogen & $H-03-036357$ & 01 & $\begin{array}{l}\text { N2 SUPPLY SYSTEM PIPING DIAGRAM } \\
\text { RADIOMET. LAB }\end{array}$ & Hand \\
\hline 327 & Nitrogen & $\mathrm{H}-03-036357$ & 02 & $\begin{array}{l}\text { N2 SUPPLY SYSTEM PIPING DIAGRAM } \\
\text { RADIOMET. LAB }\end{array}$ & Hand \\
\hline
\end{tabular}




\section{Essential Drawing List}

\begin{tabular}{|c|c|c|c|c|c|}
\hline Buirding & siston: & 8 orang & 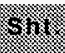 & 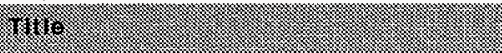 & Hod \\
\hline 327 & $\begin{array}{l}\text { Radioactive Liquid } \\
\text { Waste Sewer }\end{array}$ & $\mathrm{H}-03-047395$ & 01 & PIPING DIAGRAM RLWS LINES & Hand \\
\hline 327 & Process Sewer & $\mathrm{H}-03-047396$ & 01 & PIPING DIAGRAM RPS \& PS LINES & Hand \\
\hline 327 & HVAC Controls & $\mathrm{H}-03-049436$ & 01 & $\begin{array}{l}\text { HVAC - FMCS SENSORS INSTALILATION } \\
\text { BASEMENT PLAN }\end{array}$ & CAD \\
\hline 327. & HVAC Controls & H-03-049436 & 02 & $\begin{array}{l}\text { HVAC - FMCS SENSORS INSTALLATION IST } \\
\text { FL \& ROOF PLANS }\end{array}$ & $C A D$ \\
\hline
\end{tabular}

\begin{tabular}{|c|c|c|c|c|c|}
\hline 327 & HVAC Controls & $\mathrm{H}-03-049436$ & 03 & $\begin{array}{l}\text { HVAC - FMCS SENSORS INSTALLATION DSC } \\
\text { SCHED \& DIAGRAMS }\end{array}$ & CAD \\
\hline 327 & HVAC Controls & $H-03-049436$ & .04 & $\begin{array}{l}\text { HVAC - FMCS SENSORS INSTALLATION DSC } \\
\text { SCHED \& DIAGRAMS }\end{array}$ & CAD \\
\hline 327 & HVAC Controls & $\mathrm{H}-03-049436$ & 05 & $\begin{array}{l}\text { ELECTRICAL VENTILATION SYSTEM } \\
\text { CONTROL COMPONENTS }\end{array}$ & CAD \\
\hline 327 & Fire Alarm & H-03-058342 & 01 & $\begin{array}{l}\text { ELECTRICAL RADIO FIRE ALARM PLAN AND } \\
\text { DIAGRAM }\end{array}$ & Hand \\
\hline 327 & HVAC & $\mathrm{H}-03-070261$ & 01 & EXHAUST FLOW DIAGRAM & CAD \\
\hline 327 & Stack Monitoring & $\mathrm{H}-03-070444$ & 01 & $\begin{array}{l}\text { ISOKINETIC SAMPLER AND CABINET MAIN } \\
\text { STACK }\end{array}$ & CAD \\
\hline 327 & Stack Monitoring & $\mathrm{H}-03-070444$ & 02 & $\begin{array}{l}\text { ISOKINETIC SAMPLER AND CABINET } \\
\text { INSTALLATION SECTIONS AND DET }\end{array}$ & CAD \\
\hline 327 & Process Sewer & $\mathrm{H}-03-070466$ & 01 & RPS \& PS BELOW FIRST FLOOR PIPING & CAD \\
\hline 327 & Process Sewer & $\mathrm{H}-03-070466$ & 02 & $\begin{array}{l}\text { RPS \& PS EQPT RM \& FIRST FL PIPING } \\
\text { ISOMETRIC }\end{array}$ & CAD \\
\hline 327 & Process Sewer & $\mathrm{H}-03-070466$ & 03 & RPS \& PS PIPING DETAILS & CAD \\
\hline 327 & Process Sewer & $\mathrm{H}-03-070466$ & 04 & RPS \& PS PIPINT DETAILS & CAD \\
\hline 327 & $\begin{array}{l}\text { Radioactive Liquid } \\
\text { Waste Sewer }\end{array}$ & $\mathrm{H}-03-070467$ & 01 & RLWS BELOW FIRST FLOOR PIPING PLAN & CAD \\
\hline
\end{tabular}




\section{Essential Drawing List}

\begin{tabular}{|c|c|c|c|c|c|}
\hline Pundivg & 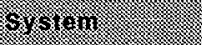 & 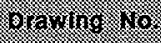 & S14 & 40 & 38 \\
\hline 327 & $\begin{array}{l}\text { Radioactive Liquid } \\
\text { Waste Sewer }\end{array}$ & $\mathrm{H}-03-070467$ & 02 & $\begin{array}{l}\text { RLWS \& MISC DRAINAGE PIPING FIRST FLOOR } \\
\text { \& BSMT ISOMETRIC }\end{array}$ & CAD \\
\hline 327 & $\begin{array}{l}\text { Radioactive Liquid } \\
\text { Waste Sewer }\end{array}$ & H-03-070467 & 03 & RLWS PIPING DETAILS & CAD \\
\hline 327 & $\begin{array}{l}\text { Radioactive Liquid } \\
\text { Waste Sewer }\end{array}$ & $\mathrm{H}-03-070467$ & 04 & RLWS PIPING DETAILS & CAD \\
\hline 327 & $\begin{array}{l}\text { Radioactive Liquid } \\
\text { Waste Sewer }\end{array}$ & $\mathrm{H}-03-070467$ & 05 & RLWS PIPING DETAILS & CAD \\
\hline 327 & $\begin{array}{l}\text { Radioactive Liquid } \\
\text { Waste Sewer }\end{array}$ & H-03-070467 & 06 & RLWS PIPING DETAILS & CAD \\
\hline 327 & Sanitary Sewer & $\mathrm{H}-03-070468$ & 01 & $\begin{array}{l}\text { SANITARY SEWER BELOW FIRST FLOOR } \\
\text { PIPING PLAN \& ISOMETRIC }\end{array}$ & \\
\hline
\end{tabular}

\begin{tabular}{|c|c|c|c|c|c|}
\hline 327 & Condensate Return & H-03-070469 & 01 & $\begin{array}{l}\text { CONDENSATE RETURN PIPING PLAN \& } \\
\text { DETAILS }\end{array}$ & CAD \\
\hline 327 & Condensate Return & H-03-070469 & 02 & $\begin{array}{l}\text { CONDENSATE RETURN PIPING EQPT RM \& } \\
\text { FIRST FL ISOMETRIC }\end{array}$ & CAD \\
\hline 327 & Condensate Return & H-03-070469 & 03 & CONDENSATE RETURN PIPING DETAILS & CAD \\
\hline 327 & Stack Monitoring & H-03-070532 & 01 & STACK SAMPLING SYSTEM FLOW DIAGRAM & CAD \\
\hline 327 & Criticality Alarm & H-03-070594 & 01 & $\begin{array}{l}\text { CRITICALITY SYSTEM CONTROL PANELS \& } \\
\text { HOWLER LOCATIONS }\end{array}$ & $C A D$ \\
\hline 327 & Criticality Alarm & $\mathrm{H}-03-070594$ & 02 & $\begin{array}{l}\text { CRITICALITY SYSTEM CONTROL PANELS \& } \\
\text { HOWLER LOCATIONS }\end{array}$ & $C A D$ \\
\hline 327 & Criticality Alarm & $\mathrm{H}-03-070594$ & 03 & $\begin{array}{l}\text { CRITICALITY SYSTEM CONTROL PANELS \& } \\
\text { HOWLER LOCATIONS }\end{array}$ & CAD \\
\hline 327 & Criticality Alarm & H-03-070594 & 04 & $\begin{array}{l}\text { CRITICALITY ALARM SYSTEM CONN ELEM } \\
\text { DIAG }\end{array}$ & CAD \\
\hline 327 & HVAC Controls & $\mathrm{H}-03-300199$ & 01 & ELECTRICAL VENTILATION CONTROL & CAD \\
\hline 327 & HVAC Controls & H-03-300199 & .02 & ELECTRICAL VENTILATION CONTROL & CAD \\
\hline
\end{tabular}




\section{Essential Drawing List}

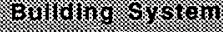

327 HVAC Controls

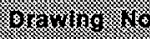

H-03-300199 sing

03
3.1.1.

ELECTRICAL VENTILATION CONTROL

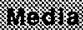

CAD

\begin{tabular}{lllll}
\hline 327 & HVAC Controls & H-03-300199 & 04 & ELECTRICAL VENTILATION CONTROL
\end{tabular}

327 HVAC Controls $\quad$ H-03-300199 05 ELECTRICAL VENTILATION CONTROL.

$327 \quad$ HVAC Controls

H-03-300199 06 ELECTRICAL VENTILATION CONTROL

CAD

327

HVAC Controls

H-03-300199

07

ELECTRICAL VENTILATION CONTROL

CAD$$
327
$$

HVAC Controls

H-03-300199

08

ELECTRICAL VENTILATION CONTROL

CAD

\begin{tabular}{lllllll}
\hline 327 & HVAC & H-03-300264 & 01 & HVAC COMPOSITE FIRST FLOOR & CAD \\
\hline
\end{tabular}

327 HVAC H-03-300265 01 SUPPLY SYSTEMS (A),(D),(E) \& (F) 1ST FLOOR CAD

\begin{tabular}{|c|c|c|c|c|c|}
\hline 327 & HVAC & $\mathrm{H}-03-300266$ & 01 & EXHAUST SYSTEMS (B) \& (G) 1ST FLOOR & CAD \\
\hline 327 & HVAC & 'H-03-300267 & 01 & HVAC COMPOSITE BASEMENT & CAD \\
\hline 327 & HVAC & $H-03-300268$ & 01 & $\begin{array}{l}\text { EXHAUST SYSTEM B CANYON EXHAUST } \\
\text { BASEMENT }\end{array}$ & CAD \\
\hline 327 & HVAC & $H-03-300269$ & 01 & $\begin{array}{l}\text { EXHAUST SYSTEM (D) CELL EXHAUST } \\
\text { BASEMENT }\end{array}$ & CAD \\
\hline 327 & HVAC & $H-03-300270$ & 01 & SECTION \& DETAILS EXHAUST SYSTEMS & CAD \\
\hline 327 & HVAC & $H-03-300271$ & 01 & $\begin{array}{l}\text { BANK OF } 9 \text { FILTERS ACTIVATED CHARCOAL } \\
\text { SYSTEM "C" EXHAUST }\end{array}$ & CAD \\
\hline 327 & Normal Power & $H-03-300284$ & 01 & $\begin{array}{l}\text { FIRST FLOOR PANEL LOCATIONS AND } \\
\text { SCHEDULES }\end{array}$ & CAD \\
\hline 327 & Normal Power & H-03-300284 & 02 & BASEMENT PANEL LOCATIONS & CAD \\
\hline
\end{tabular}




\section{Essential Drawing List}

\begin{tabular}{|c|c|c|c|c|c|}
\hline 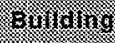 & 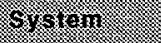 & 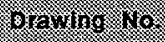 & 8314 & (4) & ringa \\
\hline 327 & Normal Power & H-03-300284 & 03 & $\begin{array}{l}\text { PANEL BOARD SCHEDULES NORMAL } \\
\text { POWER }\end{array}$ & CAD \\
\hline 327 & Normal Power & H-03-300284 & 04 & $\begin{array}{l}\text { PANEL BOARD SCHEDULES NORMAL } \\
\text { POWER }\end{array}$ & CAD \\
\hline 327 & Normal Power & $H-03-300284$ & 05 & $\begin{array}{l}\text { PANEL BOARD SCHEDULES STANDBY } \\
\text { POWER }\end{array}$ & CAD \\
\hline 327 & Normal Power & $\mathrm{H}-03-300285$ & 01 & NORMAL POWER ONE-LINE DIAGRAM & CAD \\
\hline 327 & Normal Power & $\mathrm{H}-03-300285$ & 02 & STANDBY POWER ONE-LINE DIAGRAM & CAD \\
\hline 327 & Normal Power & $H-03-300286$ & 01 & $\begin{array}{l}\text { SWITCHGEAR ELEVATION AND CUBICLE } \\
\text { ASSIGNMENT }\end{array}$ & CAD \\
\hline
\end{tabular}

\begin{tabular}{lllllll}
\hline 327 & Normal Power & H-03-300286 & 02 & ELEMENTARY DIAGRAMS & CAD \\
\hline 327 & Process Sewer & H-03-300417 & 01 & $\begin{array}{l}\text { INSTM INTCON DIAG PS EFFLUENT } \\
\text { MONITORING }\end{array}$ & CAD \\
\hline 327 & $\begin{array}{l}\text { Retention Process } \\
\text { Sewer }\end{array}$ & H-03-300417 & 02 & $\begin{array}{l}\text { INSTM INTCON DIAG RPS EFFLUENT } \\
\text { MONITORING }\end{array}$ & CAD \\
\hline 327 & Process Sewer & H-03-300421 & 01 & $\begin{array}{l}\text { INSTM/PIPING/ELEC PLANS PS \& RPS } \\
\text { EFFLUENT MONITORING }\end{array}$ & CAD \\
\hline
\end{tabular}

\begin{tabular}{lllll}
\hline 327 & Process Sewer & H-03-300422 & 01 & INSTM DETAILS PS \& RPS EFFLUENT MON
\end{tabular}

\begin{tabular}{llllll}
\hline 327 & Fire Alarm & H-03-300462 & 01 & $\begin{array}{l}\text { FIRE PROTECTION ALARM AND DETECTION } \\
\text { BASEMENT FLO }\end{array}$ & Hand \\
\hline 327 & Fire Alarm & H-03-300462 & 02 & $\begin{array}{l}\text { FIRE PROTECTION ALARM AND DETECTION } \\
\text { FIRST FLOOR }\end{array}$ & Hand \\
\hline 327 & Fire Alarm & H-03-300462 & 03 & $\begin{array}{l}\text { FIRE PROTECTION ALARM \& DETECTION SPR } \\
\text { PLAN, DETA }\end{array}$ \\
\hline 327 & Fire Alarm & H-03-300463 & 01 & $\begin{array}{l}\text { FIRE PROTECTION ALARM \& DETECION FCP. Hand } \\
\text { WIRING DIAG }\end{array}$ \\
\hline 327 & Fire Alarm & H-03-300463 & 02 & $\begin{array}{l}\text { FIRE PROT ALARM \& DETECTION DETAILS } \\
\text { AND DIAGRAMS }\end{array}$ & Hand \\
\hline
\end{tabular}




\section{Essential Drawing List}

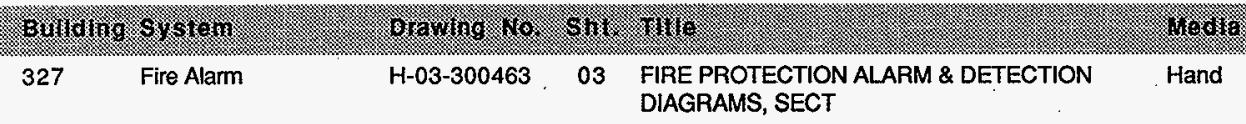

\begin{tabular}{|c|c|c|c|c|c|}
\hline 327 & $\begin{array}{l}\text { Radioactive Liquid } \\
\text { Waste Sewer }\end{array}$ & $\mathrm{H}-03-301513$ & 02 & SUMP PUMP MODIFICATIONS & $\mathrm{CAD}$ \\
\hline 327 & $\begin{array}{l}\text { Radioactive Liquid } \\
\text { Waste Sewer }\end{array}$ & $\mathrm{H}-03-301513$ & 03 & SUMP PUMP MODIFICATIONS & CAD \\
\hline 327 & $\begin{array}{l}\text { Radioactive Liquid } \\
\text { Waste Sewer }\end{array}$ & $\mathrm{H}-03-301513$ & 04 & SUMP PUMP MODIFICATIONS & CAD \\
\hline 327 & $\begin{array}{l}\text { Radioactive Liquid } \\
\text { Waste Sewer }\end{array}$ & H-03-301513 & 05 & SUMP PUMP MODIFICATIONS & CAD \\
\hline 327 & $\begin{array}{l}\text { Safety Showers \& } \\
\text { Eye Wash }\end{array}$ & $H-03-301566$ & 01 & $\begin{array}{l}\text { EMERGENCY SHOWER, EYEWASH } \\
\text { FLUSHLINE BASEMENT MODS }\end{array}$ & CAD \\
\hline 327 & $\begin{array}{l}\text { Safety Showers \& } \\
\text { Eye Wash }\end{array}$ & $\mathrm{H}-03-301566$ & 02 & $\begin{array}{l}\text { EMERGENCY SHOWER, EYEWASH } \\
\text { FLUSHLINE FIRST FLOOR MODS }\end{array}$ & CAD \\
\hline 327 & $\begin{array}{l}\text { Safety Showers \& } \\
\text { Eye Wash }\end{array}$ & $H-03-301566$ & 03 & $\begin{array}{l}\text { EMERGENCY SHOWER, EYEWASH } \\
\text { FLUSHLINE MOD DETAILS }\end{array}$ & CAD \\
\hline 327 & $\begin{array}{l}\text { Radioactive Liquid } \\
\text { Waste Sewer }\end{array}$ & $\mathrm{H}-03-301783$ & 01 & $\begin{array}{l}\text { ELECTRICAL DETAILS DIAGRAMS BLDG } \\
\text { RLWS }\end{array}$ & Hand \\
\hline 327 & $\begin{array}{l}\text { Radioactive Liquid } \\
\text { Waste Sewer }\end{array}$ & $\mathrm{H}-03-301784$ & 01 & $\begin{array}{l}\text { ELECTRICAL ANNUNCIATOR DIAGRAM AND } \\
\text { SCHEDULE }\end{array}$ & Hand \\
\hline 327 & Radiation Alarm & $\mathrm{H}-03-301786$ & 01 & $\begin{array}{l}\text { INSTRUMENTATION RADIATION AREA } \\
\text { MONITORS FIRST FLOOR }\end{array}$ & $C A D$ \\
\hline 327 & Radiation Alarm & $H-03-301786$ & 02 & $\begin{array}{l}\text { INSTRUMENTATION RADIATION AREA } \\
\text { MONITORS BASEMENT }\end{array}$ & $C A D$ \\
\hline 327 & Radiation Alarm & $\mathrm{H}-03-301786$ & 03 & $\begin{array}{l}\text { INSTRUMENTATION RADIATION AREA } \\
\text { MONITORS DETAILS }\end{array}$ & CAD \\
\hline 327 & Sanitary Water & $\mathrm{H}-04-050217$ & 01 & PLAN \& SECTIONS MAIN SERVICE PIPING & Hand \\
\hline 327 & Sanitary Water & $\mathrm{H}-04-050217$ & 02 & PLAN \& SECTIONS MAIN SERVICE PIPING & Hand \\
\hline 327 & Vacuum Air Sampling & $H-04-050218$ & 01 & AIR SAMPLING PIPING & Hand \\
\hline
\end{tabular}




\section{Essential Drawing List}

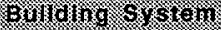

327 Sanitary Water

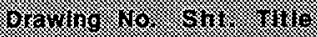

$\mathrm{H}-04-050220$
01
FLOW DIAGRAMS
4.6.

Hand

\begin{tabular}{|c|c|c|c|c|c|}
\hline 327 & Sanitary Water & $H-04-050223$ & 01 & EQUIPMENT ROOM LAYOUT \& DETAILS & Hand \\
\hline 327 & HVAC Controls & $H-04-050238$ & 01 & $\begin{array}{l}\text { DIAGRAMS OF CONTROL INSTRUMENTS } \\
\text { VALVES \& DAMPERS }\end{array}$ & Hand \\
\hline 327 & Steam & $\mathrm{H}-04-050239$ & 01 & $\begin{array}{l}\text { STEAM \& CONDENSATE PIPING EQUIPMNT } \\
\text { ROOM }\end{array}$ & Hand \\
\hline 327 & Steam & $H-04-050240$ & 01 & $\begin{array}{l}\text { STEAM \& CONDENSATE PIPING FOR } \\
\text { BOOSTER COILS }\end{array}$ & Hand \\
\hline 327 & Normal Power & $\mathrm{H}-04-050242$ & 01 & SINGLE LINE DIAGRAM & Hand \\
\hline 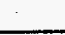 & ; & & & & \\
\hline 327 & Normal Power & $H-04-050243$ & 01 & SUBSTATION \& MAIN SWITCH GEAR & Hand \\
\hline 327 & HVAC Controls & $\mathrm{H}-04-050246$ & 01 & $\begin{array}{l}\text { ELECTRICAL VENTILATION SYSTEM } \\
\text { ELEMENTARY DIAGRAMS }\end{array}$ & CAD \\
\hline 327 & HVAC Controls & $\mathrm{H}-04-050246$ & 02 & $\begin{array}{l}\text { ELECTRICAL VENTHLATION SYSTEM } \\
\text { ELEMENTARY DIAGRAMS }\end{array}$ & CAD \\
\hline 327 & HVAC Controls & $\mathrm{H}-04-050246$ & 03 & $\begin{array}{l}\text { ELECTRICAL VENTILATION SYSTEM } \\
\text { CONTROL PANEL. }\end{array}$ & CAD \\
\hline 327 & Normal Lighting & $\mathrm{H}-04-050254$ & 01 & PANEL SCHEDULES \& DETAILS & Hand \\
\hline 327 & Normal Power & $\mathrm{H}-04-050257$ & 01 & MISC WIRING DIAGRAMS & Hand \\
\hline
\end{tabular}

